\title{
Morphological and Transcriptomic Comparison of Adipose and Bone Marrow Derived Porcine Stem Cells
}

\author{
Elisa Monaco ${ }^{1,2, \#}$, Alecsandra Sobreira de Lima, ${ }^{1, \#}$, Massimo Bionaz ${ }^{1}$, Aaron Maki², \\ Shanna M. Wilson ${ }^{1,2}$, Walter L. Hurley ${ }^{1}$, and Matthew B. Wheeler ${ }^{1,2, *}$ \\ ${ }^{1}$ Department of Animal Sciences, Laboratory of Stem Cell Biology and Engineering and ${ }^{2}$ Institute for Genomic Biology, \\ University of Illinois at Urbana-Champaign, Urbana, Illinois, USA
}

\begin{abstract}
In the present study we provided a morphological and transcriptomic comparison of adult porcine adiposederived stem cells (ADSC) and bone marrow-derived stem cells (BMSC) as they differentiated in vitro towards the osteogenic and adipogenic lineages for up to 4 weeks. The long term goal of this comparison is to assess the possibility of using ADSC as a potential alternative to BMSC as a source of autologous adult stem cells in human therapies.

Our data indicated that ADSC can differentiate into osteocytes and adipocytes similar to BMSC but with some differences. During the osteogenic differentiation both cell types went through morphological changes; however, while ADSC formed predominately osteogenic islands (nodules) in the culture dish, BMSC formed a continuous osteogenic sheet of small nodules. Transcriptomic analysis revealed that both cell types responded to the osteogenic induction. However, BGLAP mRNA expression did not increase in ADSC suggesting, together with the percentage area stained observed for Alizarin Red and von Kossa in ADSC, a lesser mineralization of bone matrix in this cell type compared to BMSC.

During the adipogenic induction ADSC as well as BMSC were able to achieve the morphological and transcriptome changes characteristic of the adipogenic lineage. After 7 days of differentiation the expression patterns of DGAT2 and $A D F P$ became greater in ADSC versus BMSC, which agreed with the larger lipid droplets formation observed in the ADSC by Oil Red O staining.

Our findings represent an important step towards the assessment of using ADSC as an alternative to BMSC in therapeutic applications.
\end{abstract}

\section{INTRODUCTION}

Degenerative diseases like osteoporosis and arthritis, as well as traumatic injuries, including non-union fractures and tumor resections, pose significant challenges for health care professionals. Therapeutic use of mesenchymal stem cells (MSC) is one potential solution, which has already been employed in clinical trials, including the treatment of human myocardial infarction, osteogenesis imperfecta, and graft versus host disease [1-3]. Mesenchymal stem cells can differentiate into a wide variety of cell types including osteoblasts [4], adipocytes [5], myoblasts [6], chondrocytes [7], and neurons [8]. Mesenchymal stem cells also display several anti-inflammatory properties, presenting the possibility for allogenic transplantation [9]. Therefore, this cell population might have immense potential for a diverse range of clinical applications.

Historically, MSC were first isolated from the bone marrow (BMSC) [10]. Although bone marrow is the current gold standard tissue source for therapy, stem cells capable of differentiating into mesenchymal lineages and even into the neurogenic phenotypes, also have been isolated from adipose

*Address correspondence to this author at the Institute for Genomic Biology, University of Illinois at Urbana-Champaign, Urbana, Illinois, USA; Tel: (217)-333-2239; Fax: (217) 333-8286;

E-mail: mbwheele@illinois.edu

\#These authors contributed equally to this work. tissue (ADSC) [11, 12]. Adipose has several advantages as a tissue source, including a higher stem cells concentration compared to bone marrow [13], easy accessibility, and abundance $[11,12]$. In fact, adipose tissue is distributed in large amount throughout the body and it is readily available due to procedures such as liposuction and lipectomy. The clinical value of bone marrow for treating diseases such as leukemia already makes it a valuable tissue [14]. Worldwide, an estimated one million liposuctions are performed annually [15], generating anywhere from $100 \mathrm{~mL}$ to $>3 \mathrm{~L}$ of lipoaspirate tissue [16] which is usually discarded. These factors make ADSC a viable potential alternative to BMSC for clinical therapy. Moreover, the possibility of using ADSC, as an alternative to BMSC for clinical therapy in humans, would relieve the patients from a very painful surgery such as the bone marrow aspiration, which can result in high donor site morbidity including discomfort at the harvest site, fatigue, and pain in the pelvic bones [17].

However, before this cell source substitution can be applied in human clinical settings, the morphological and functional properties of stem cells, derived from adipose tissue and bone marrow, and their differentiated progeny must be compared. The use of an animal model, such as swine, that adequately simulates the structure and physiology of humans, provides a readily available source of mesenchymal stem cells for research, and allows for improvements in therapeutic protocols [18-20]. Pigs have been used as a large 
animal model for studying human clinical therapies as diverse as heart surgery [18], genetic disorders [21], and drug testing [22]. In addition, pigs have ample subcutaneous fat which is similar to that in humans.

The purpose of the present study was to characterize and directly compare, by histological staining and real time RTPCR analysis, the temporal in vitro differentiation of porcine ADSC and BMSC towards the osteogenic and adipogenic lineages. The basic characteristics of these cell populations are determined to aid in the comparison with human ADSC and BMSC under similar conditions.

\section{MATERIALS AND METHODOLOGY}

\subsection{ADSC Isolation and Culture In Vitro}

Subcutaneous back fat and bone marrow were acquired from 3 castrated Yorkshire crossbred male pigs, at approximately 6 months of age, under protocols approved by the University of Illinois Institutional Animal Care and Use Committee (IACUC). Pigs were euthanized at the University of Illinois Meat Science Laboratory abattoir. The skin overlying the loin area was shaved to remove the hair and scrubbed three times using Betadine ${ }^{\circledR}$ solution (Povidoneiodine, $10 \%$ - Purdue Products L.P., Cranbury, NJ), and three times with $70 \%$ ethanol (Aaper Alcohol and Chemical Co. Shelbyville, KY) to avoid contamination of the sample. A square of subcutaneous back fat $\left(\sim 100 \mathrm{~cm}^{2}\right)$ was then excised from the sanitized area from each pig, placed in a sterile plastic bag, transported to the laboratory on ice and placed at $4^{\circ} \mathrm{C}$ until cell harvest $(<1 \mathrm{hr})$. The subcutaneous fat was dissected from the skin with a sterile scalpel. All the surfaces of the fat, that were originally exposed at tissue harvest, were also trimmed off with a sterile scalpel blade so the only sterile fat was processed for cell isolation. Strips of sterile fat were washed twice in Dulbecco's Phosphate Buffer Saline (DPBS, Sigma Aldrich D5773, St. Louis, MO) containing 1\% Penicillin G-Streptomycin (Sigma P3539) and $5.0 \mathrm{mg} / \mathrm{L}$ of Amphotericin B (Sigma A9528). After washing, tissue was minced with scalpel blades and then digested with $0.075 \%$ collagenase type I-A (Sigma C2674) in DPBS, in a $50 \mathrm{~mL}$ conical tube (Corning, NY) (v/v - tissue/collagenase), in the incubator at $37^{\circ} \mathrm{C}$ for $90 \mathrm{~min}$. The conical tubes, containing fat and collagenase, were vigorously shaken every 10 to $15 \mathrm{~min}$ to ensure a uniform digestion. After digestion, tubes were centrifuged at $200 \times \mathrm{g}$ for $10 \mathrm{~min}$ at room temperature. The buoyant cell fraction and supernatant were discarded and $2 \mathrm{~mL}$ of red blood cell lysis buffer (Sigma R7757) was added to the pellet and gently mixed for 2 min. Subsequently, $20 \mathrm{~mL}$ of DPBS were added to the tubes and were centrifuged at $200 \times \mathrm{g}$ for $5 \mathrm{~min}$ at room temperature, to obtain a cell pellet that was then re-suspended in culture medium. The culture medium used was high glucose Dulbecco's Modified Eagle's Medium (DMEM, Sigma D5648), supplemented with 10\% Fetal Bovine Serum (FBS, BenchMark $^{\mathrm{TM}}$, Gemini Bio -Products, West Sacramento, CA), plus $1 \%$ Penicillin G-Streptomycin and $5.0 \mathrm{mg} / \mathrm{L}$ of Amphotericin B. Cells were counted using an hemocytometer, plated in $75 \mathrm{~cm}^{2}$ Corning cell culture flasks at $7.5 \times 10^{5}$ cells in $15 \mathrm{~mL}$ of culture medium, and incubated at $39^{\circ} \mathrm{C}$ and $5 \%$ $\mathrm{CO}_{2}$ in $100 \%$ humidified air. Medium was changed every other day until the initial cell culture passage. Passage 0 cells reached confluence at approximately day 10 of culture. In order to keep the cells at a sufficiently low density to stimulate further growth, the initial cell cultures were washed using DPBS and harvested by digestion with $0.25 \%$ Trypsin (Sigma T4799) - 0.04\% EDTA (Sigma E6753) for 3 min. Trypsin was then inactivated by adding an equivalent volume of culture medium and the cells were centrifuged at 200 $\times \mathrm{g}$ for $5 \mathrm{~min}$ at room temperature. Cells were resuspended in culture medium for plating in $75 \mathrm{~cm}^{2}$ cell culture flasks at a density of approximately $7.5 \times 10^{5}$ cells $/ 75 \mathrm{~cm}^{2}$. These passage 1 cells were $80 \%$ confluent after 4 days. Cells were trypsinized, as described above, and frozen at $3 \times 10^{6}$ cells per $1.2 \mathrm{~mL}$ in cryogenic vials (Nalgene ${ }^{\circledR}$ Labware, Rochester, NY). Freezing medium consisted of $75 \%$ DMEM supplemented with 15\% FBS and $10 \%$ dimethyl sulfoxide (DMSO, Sigma D2650). Vials were placed in Nalgene Cryo $1^{\circ} \mathrm{C}$ Freezing containers (Nalgene ${ }^{\circledR}$ Labware) and placed in a $-80^{\circ} \mathrm{C}$ freezer. On the following day, cells were transferred to liquid nitrogen and stored until further use.

\subsection{BMSC Isolation and Culture In Vitro}

Intact femurs were removed, at slaughter, from the same 3 barrows used to harvest ADSC. Femurs were placed in sterile plastic bags, transported to the laboratory and placed at $4^{\circ} \mathrm{C}$ until cell harvest $(<1 \mathrm{hr})$. In the laboratory, the remaining soft tissue around the femur was removed using a scalpel blade. The bone was rinsed with $20 \%$ bleach followed by deionized water and dried before being cut. The femur was secured in a vise and cut with a sterile hand saw at the epiphyses on one end only. This was done using sterile instruments to ensure that marrow remained sterile. Bone marrow was extracted under a laminar flow hood. Marrow was scooped out of the bone using a sterile spatula, and placed into a sterile glass dish containing DPBS with $1 \%$ Penicillin G-Streptomycin and $5.0 \mathrm{mg} / \mathrm{L}$ of Amphotericin B. Tissue was minced using a scalpel blade and placed into a 50 $\mathrm{mL}$ Corning conical tube and digested as described for ADSC. All the following steps, up to freezing of the cells, were the same as described for the ADSC, except for the concentration of the cells at plating that in this case was 1.6 $\times 10^{6}$ cells in $15 \mathrm{~mL}$ culture medium.

\subsection{Colony Forming Unit Assay}

For determination of colony forming units (CFU), ADSC and BMSC were harvested from porcine bone marrow and subcutaneous adipose tissue as described previously. Harvested ADSC were plated at a density of 25,000 cells $/ 25 \mathrm{~cm}^{2}$ culture flask, and BMSC were plated at a density of 100,000 cells $/ 25 \mathrm{~cm}^{2}$ culture flask. After plating the cells were incubated at $39^{\circ} \mathrm{C}$ and $5 \% \mathrm{CO}_{2}$ for 9 days with media changes occurring every 48 hours. After 9 days of culture, colonies were fixed using formalin for 30 minutes, exposed to hematoxylin staining solution for $30 \mathrm{~min}$, and washed with tap water. ADSC and BMSC colonies were then counted using an inverted microscope (Nikon Diaphot microscope). Cell clusters consisting of at least 20 cells were classified as a CFU colony.

\subsection{ADSC and BMSC Osteogenic and Adipogenic Dif- ferentiation In Vitro}

Prior to differentiation studies, frozen ADSC and BMSC were thawed and plated (passage 2) at $7.5 \times 10^{5}$ cells $/ 75 \mathrm{~cm}^{2}$ 
in $15 \mathrm{~mL}$ supplemented culture medium. The cells were cultured for 4 days and then trypsinized, and viable cells counted and plated (passage 3 ) at the same density of before. Cells from each pig were cultured separately.

For the cell staining, cells were plated at $2.5 \times 10^{5}$ cells $/ 25 \mathrm{~cm}^{2}$ culture flask in $5 \mathrm{~mL}$ supplemented culture medium. For mRNA isolation and reverse transcriptase RTPCR, cells were plated at $7.5 \times 10^{5}$ cells $/ 75 \mathrm{~cm}^{2}$ in culture flask in $15 \mathrm{~mL}$ supplemented culture medium.

Cells were incubated for 4 days to reach $80 \%$ confluence prior starting the differentiation treatment. To induce the differentiation of ADSC and BMSC into osteogenic or adipogenic cells in vitro, ADSC and BMSC were cultured with specific osteogenic or adipogenic differentiation medium. Osteogenic medium consisted of basic high glucose DMEM supplemented with $100 \mathrm{nM}$ dexamethasone (Sigma D4902), $10 \mathrm{mM} \beta$-glycerophosphate (Sigma G6376), $0.05 \mathrm{mM}$ ascorbic acid-2-phosphate (Sigma A4403), 10\% FBS, 1\% Penicillin G-Streptomycin, and $5.0 \mathrm{mg} / \mathrm{L}$ Amphotericin B. Adipogenic medium consisted of basic high glucose DMEM supplemented with $1.0 \mu \mathrm{M}$ dexamethasone, $0.5 \mathrm{mM}$ isobutylmethylxanthine (Sigma I5879), $10 \mu \mathrm{M}$ insulin (Sigma I9278), $200 \mu \mathrm{M}$ indomethacin (Sigma I7378), 10\% FBS, 1\% Penicillin G-Streptomycin, and $5.0 \mathrm{mg} / \mathrm{L}$ Amphotericin B.

Cells were cultured in these media for 4 weeks with media changed every 3 days.

\subsection{Histological Staining for Osteogenic and Adipogenic Differentiation}

At differentiation day (dd) $0,2,4,7,14,21$, and 28 cells were rinsed with DPBS and stained with the osteogenic histological stains Alizarin Red S, Alkaline Phosphatase, and von Kossa, and the adipogenic histological stain Oil Red O. Details on the staining procedure are reported in Suppl. Materials.

\subsection{RNA Extraction, Quantitative Real-Time RT-PCR (qPCR), Primer Design and Evaluation, and Selection of the Internal Control Genes for qPCR Normalization}

At dd $0,2,4,7,14,21$, and 28 cells were trypsinized as described above and total RNA was immediately isolated using the RNeasy ${ }^{\circledR}$ Mini Kit (Qiagen Sciences, Germantown, $\mathrm{MD}$ ) according to the manufacturer's protocol. Any residual genomic DNA was eliminated by treatment with RNase-Free DNase Set $^{\circledR}$ (Qiagen). RNA concentration was measured with a NanoDrop ND-1000 spectrophotometer (NanoDrop Technologies, Wilmington, DE, http://www.nanodrop.com). The purity of RNA (A260/A280) was > 1.90. Details for qPCR, primer design, primer evaluation, and selection and evaluation of the internal control genes (ICG) for qPCR normalization are reported in Suppl. Materials.

The qPCR data were normalized using the geometric mean of 3 or 4 internal control genes (GAPDH, GTF2H3, $N U B P 1$, and $P P P 2 C B$ ). The normalized data were transformed in $n$-fold change relative to dd 0 for each cell type and differentiation. To estimate standard errors at $\mathrm{dd} 0$, and prevent biases in statistical analysis, normalized data were transformed to obtain a perfect average of 0 at dd 0 , leaving the proportional difference between the biological replicates.
The same proportional change was calculated at all the other time points, inside the same cell type and differentiation, to obtain a $n$-fold change relative to dd 0 .

Normalized data nontransformed as $n$-fold change were also used to assess similarity in mRNA abundance of selected genes between the two cell types on dd 0 and during differentiation (dd 2, 4, 7, 14, 21, 28).

To assess percentage mRNA abundance between measured genes in each cell type, the median relative percentage abundance of transcript for measured genes, was calculated by the inverse of median $\mathrm{Ct}$ value of all samples and corrected by the efficiency of PCR amplification as previously reported [23]. This analysis was necessary to compare percentage mRNA abundance between genes in the same cell, since the PCR data were obtained by using a standard curve (relative quantification), which precluded a direct comparison in abundance among different transcripts.

\subsection{Selection of Osteogenic and Adipogenic Genes}

Transcript abundance of biochemical markers of bone turnover such as alkaline phosphatase $(A L P)$, bone gammacarboxyglutamate (gla) protein (osteocalcin) (BGLAP), collagen type I alpha 1 (COLIAI), secreted protein acidic cysteine-rich (osteonectin) (SPARC), and secreted phosphoprotein 1 (osteopontin) (SPP1), were used to characterize the osteogenic differentiation of ADSC and BMSC. ALP is an early osteoblast marker as is SPARC, while BGLAP is characteristic of the late stages of osteoblastic differentiation [24, 25]. COL1A1 is the major extracellular bone protein [25]. $S P P 1$ is responsible for the regulation of the formation and remodeling of mineralized tissue [26].

Genes selected for the adipogenic differentiation were acyl-CoA synthetase long-chain family member 1 (ACSL1), adipose differentiation related protein (adipophilin) $(A D F P)$, adiponectin $(A D I P O Q)$, CD36 molecule (thrombospondin receptor) (CD36), CCAAT/enhancer binding protein (C/EBP) alpha (CEBPA), diazepam binding inhibitor (acylCoA-binding protein) $(D B I)$, diacylglycerol O-acyltransferase homolog 2 (DGAT2), peroxisome proliferator-activated receptor gamma $(P P A R G)$, and stearoyl-CoA desaturase (delta-9-desaturase) ( $S C D) . A D F P$ is a lipid droplet protein and it is considered an early marker of adipogenic differentiation [27, 28]. Adiponectin is almost exclusively secreted from adipose tissue and its gene $(A D I P O Q)$ is under the control of $C E B P A$ which activates its transcription and $P P A R G$ which inhibits it [29]. CD36 and ACSL1 are proteins implicated in free fatty acid influx [30]. CEBPA and PPARG coordinate adipocyte differentiation in vitro [31]. DBI responds to lipogenic transcription factors and is involved in lipid metabolism pathways [32]. DGAT2 and $S C D$ are involved in triacylclycerol synthesis [33]. Features of the primer pairs for the 14 selected differentiation gene markers and for the ICG are shown in Table $\mathbf{S 1 .}$

\subsection{Image Analysis}

Pictures (magnification 100 or $400 \times$ ) of ADSC and BMSC differentiated into osteogenic and adipogenic lineages were analyzed and pixels were measured using the image analysis program ImageJ (U.S. National Institutes of Health). Stained area was determined by using a color deconvolution 
algorithm [34]. Further details are reported in Suppl. Materials.

\subsection{Statistical Analysis}

Gene expression and outcome of image analysis were performed using a mixed model of SAS (release 8.0; SAS Inst.,Cary, NC) with repeated time. Spatial power was the most appropriate covariate structure for repeated measures analysis for the present experiment. The assessment of goodness of fit was performed using Akaike's Information Criterion. The model included fixed effect of time (dd) and cell type (ADSC and BMSC), and interaction $\mathrm{dd} \times$ cell type. Pig $(n=3)$ was considered a random variable. Before statistical analysis, data were assessed for normal distribution using proc univariate normal of SAS with Shapiro Wilk (W) and Kolmogorov-Smirnov (D) tests. Data were considered normal distributed when $\mathrm{P}$-value of $\mathrm{W}$ and $\mathrm{D}$ were $>0.01$. If data were no-normal distributed, log or square root transformations were used to obtain a P-value of $\mathrm{W}$ and $\mathrm{D}>0.01$. In graphs are shown LS-means from SAS and LS-means back transformed when used normalized data. Due to the impossibility of back-transforming the SEM from log-transformed data we decided to omit the SEM from the graphs. The comparison in mRNA abundance at dd 0 and number of CFU were analyzed by GLM model of SAS, with cell type as fixed effect and pig as random effect. Differences in relative expression were discussed when there was a time $\times$ cell type interaction effect of $\mathrm{P} \leq 0.05$ and differences at each time point between cell types were considered significant when Pdiff $\leq 0.05$.

\section{RESULTS}

\subsection{Primary Culture and Colony Forming Unit}

On the first day of culture, after cells were isolated from adipose tissue or bone marrow, the population consisted of round-shaped erythrocytes and non adherent cells. By day 23 , cells adhered to the culture flask (Fig. 1), exhibiting a stretched out fibroblast-like shape, and non adherent cells were removed by medium exchange. On day 10 of culture, the cells were $60-80 \%$ confluent, organized in colonies, and presented a long-spindle-shaped fibroblast phenotype (Fig. 1).

After 9 days of culture, from 25,000 ADSC plated $390 \pm$ $0.02(0.39 \pm 0.02 \%$; mean $\pm \mathrm{SEM})$ colonies were formed, and from 100,000 BMSC plated $70 \pm 0.01(0.07 \pm 0.01 \%)$ colonies were formed.

\subsection{Osteogenic Differentiation}

On dd 0, ADSC were fibroblast-like cells (Fig. 2). On dd 2 after the induction of osteogenic differentiation, cells were elongated but in a cubical-polygonal shape. On dd 4, some cells were oriented forming clusters. Until dd 4 cells did not stain positively by any histological staining method. On dd 7 , multilayer colonies started to form and stained positively for Alkaline Phosphatase (Figs. 2 and 3). Also calcification started as demonstrated by the presence of red nodules stained with Alizarin Red S, as well as yellowish-brown nodules stained with von Kossa (Figs. 2 and 3). The cells that remained close to the nodules, but did not participate in the nodules formation, were still elongated and in monolayer

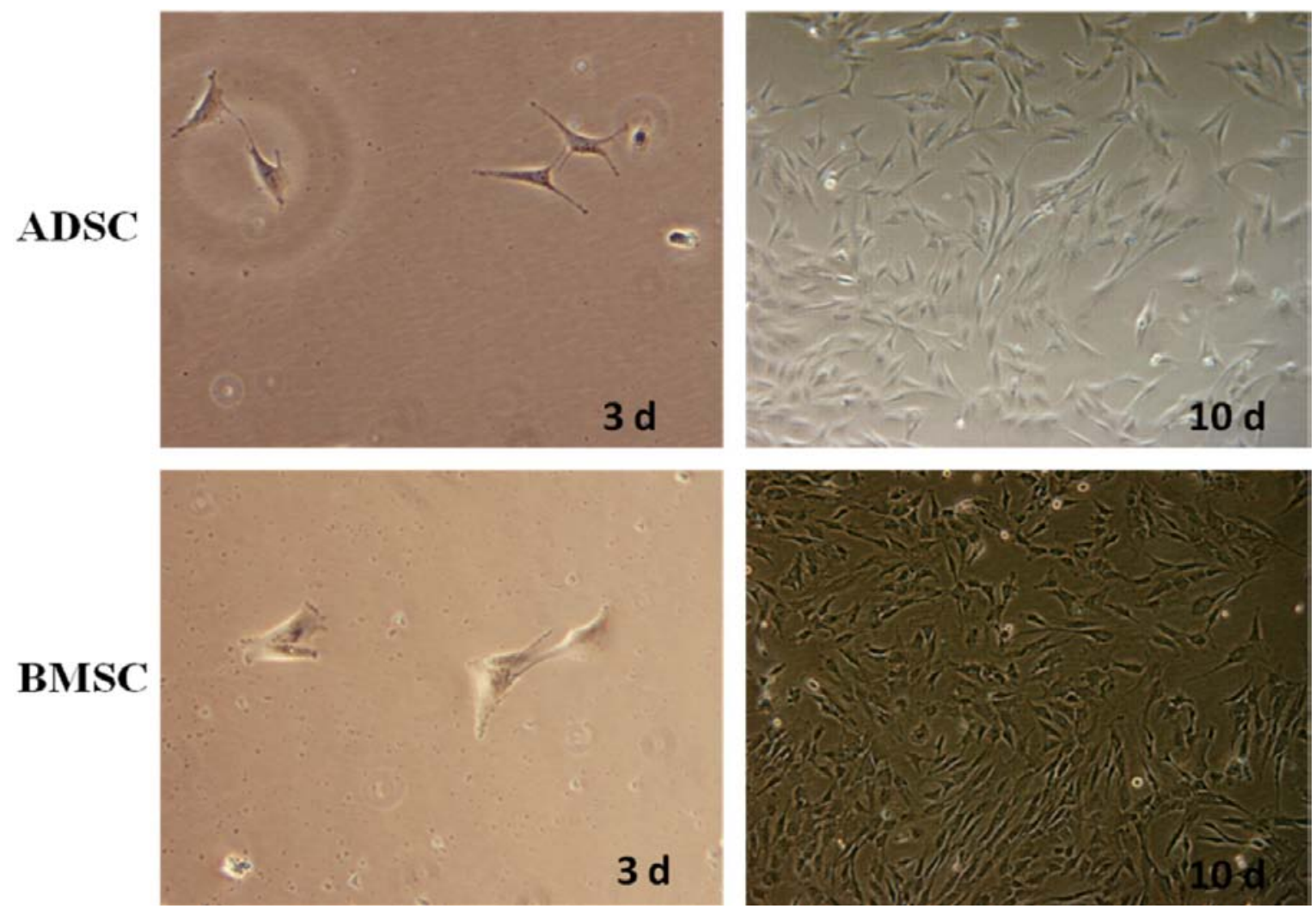

Fig. (1). Primary in vitro culture of ADSC and BMSC. Panels are (from left to right and top to bottom) adherent ADSC after 3 days (d) of culture and $60 \%$ confluent ADSC after $10 \mathrm{~d}$ of culture; adherent BMSC after $3 \mathrm{~d}$ of culture and $80 \%$ confluent BMSC after $10 \mathrm{~d}$ of culture. Magnification is $400 \times$ for left panels top and bottom and $100 \times$ for right panels top and bottom. 


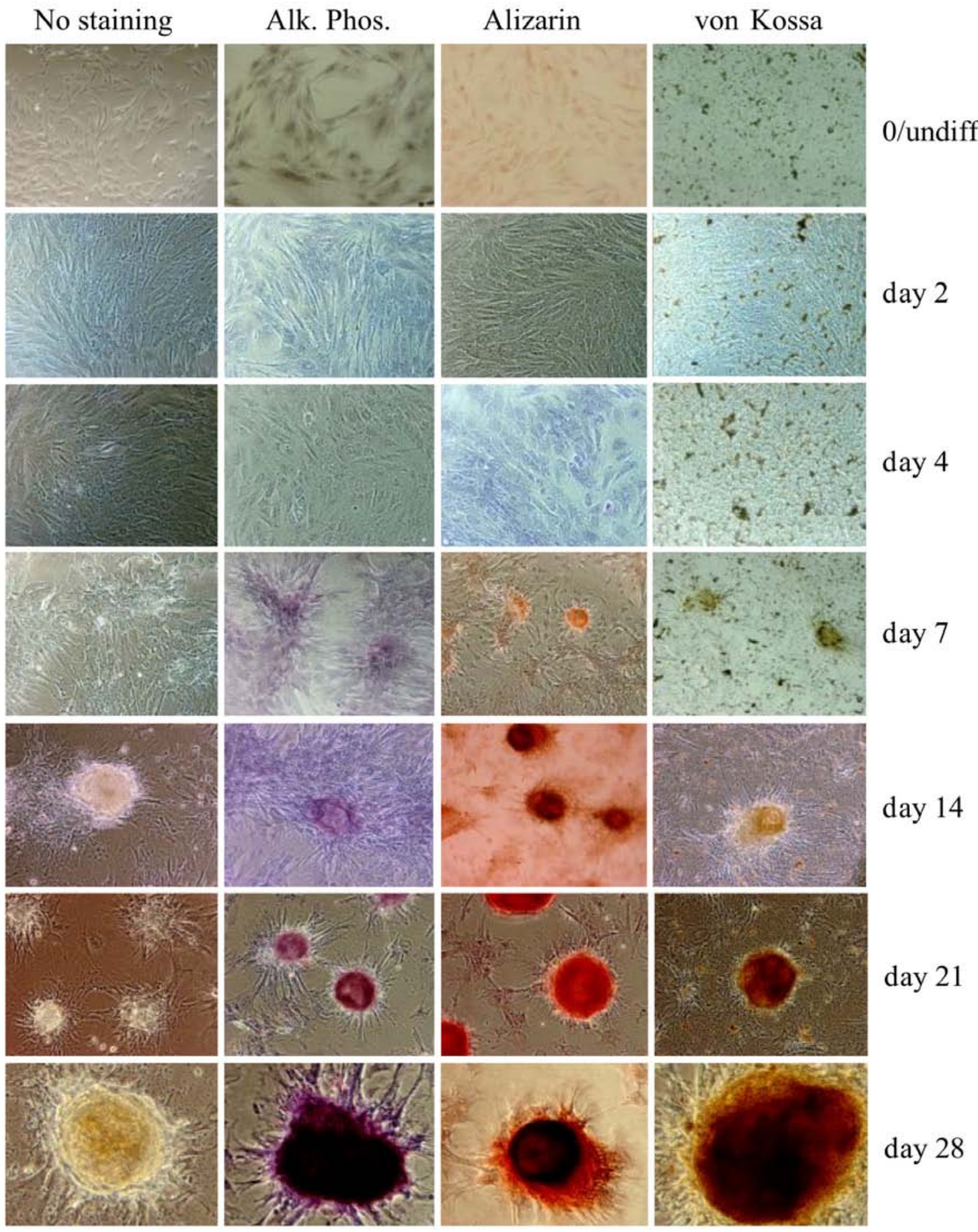

Fig. (2). Morphological changes in ADSC during osteogenic differentiation in vitro. Panels are staining for (left to right): no staining, Alkaline Phosphatase (Alk. Phos.; cells with Alk. Phos. appear red), Alizarin Red S (positive cells containing calcium deposits appear red), and von Kossa (black areas are areas of calcium deposition). Panels are days of differentiation (top to bottom): undifferentiated (day 0), and days of differentiation 2, 4, 7, 14, 21, and 28. Passage 3 cells were induced to differentiate by osteogenic medium at $\sim 80 \%$ confluence. Magnification is $100 \times$ for all the pictures except for dd 28 for all staining and dd 0 for Alkaline Phosphatase staining which are $400 \times$. Biological replicates $=3$.

with absence of positive staining. On dd 14, new clusters of cells started to form, while the nodules already formed increased numerically in size (Fig. S6) and became more defined. The cells surrounding the nodules were stained positively for Alkaline Phosphatase, while the cells farther from the nodules remained in monolayer and did not stain. On dd 21, we observed larger nodules compared to dd 7 (Fig. S6), much more defined, and clearly stained positively for all 3 histological staining (Figs. 2 and 3). On dd 21, we also observed nodules of new formation. On dd 28, the nodules reached, in average, a radius of $>37 \mu \mathrm{M}$ (Fig. S6).

BMSC on dd 0 were fibroblastic-like cells (Fig. 4). On dd 2, morphological changes occurred in some of the BMSC, which changed their shape from elongated to polygonal (Fig. 
4). On dd 4, most of the cells were polygonal and covered the whole bottom of the flask forming areas of densely accumulated cells. Until dd 4, cells did not stain positively for any of the histological stains. On dd 7, clumps of cells
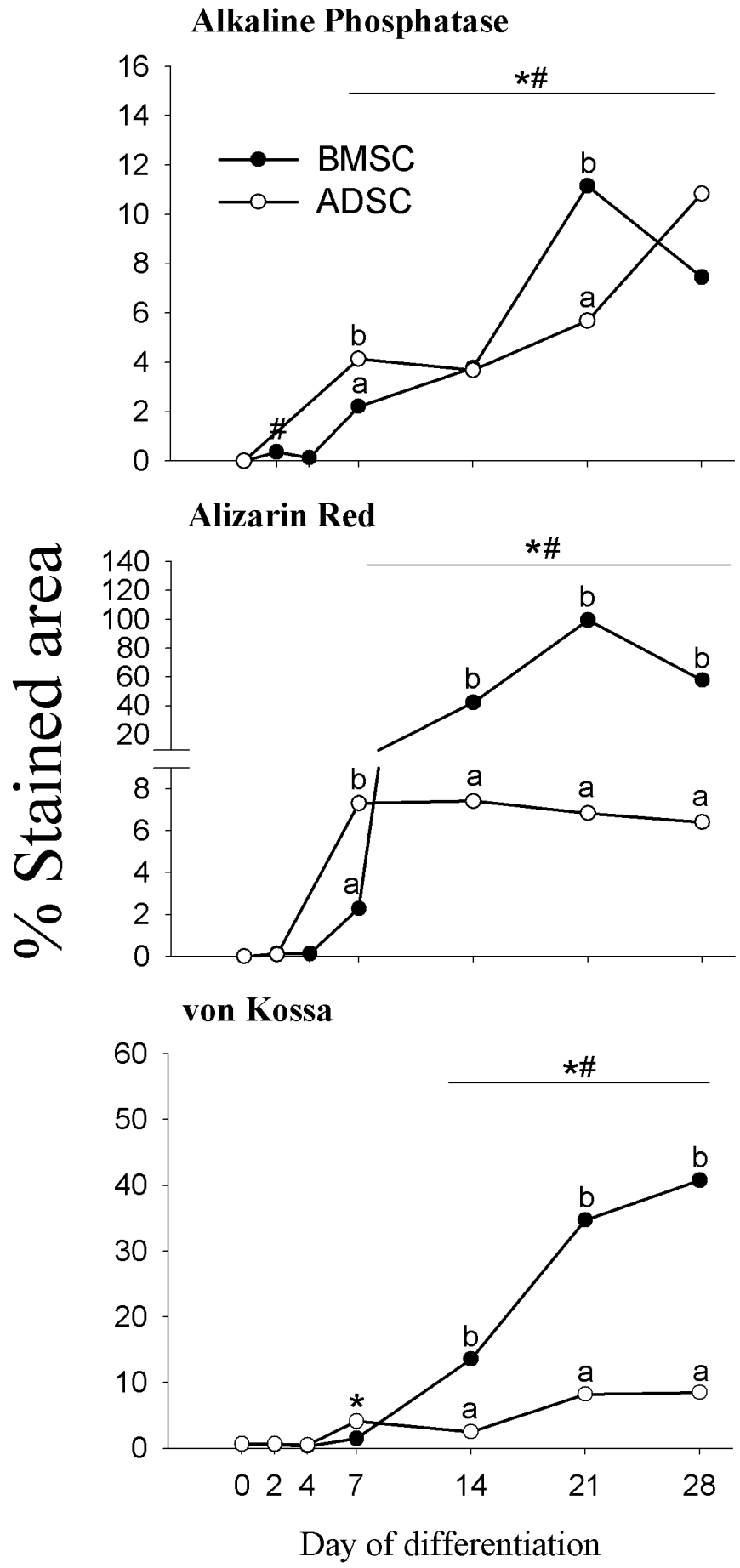

Fig. (3). Percentage (\%) area occupied by stained ADSC and BMSC during osteogenic differentiation in vitro. Data are expressed as the area stained by Alkaline Phosphatase. Alizarin Red $\mathrm{S}$, and von Kossa as a percentage of total area. Superscripts denote: *, \# is $\mathrm{P}<0.05$ at each time point vs. dd 0 in ADSC and BMSC, respectively; ${ }^{\mathrm{a}, \mathrm{b}}$ is $\mathrm{P}<0.05$ between cell types at individual time points. ${ }^{a}$ is used for significantly lower values, while ${ }^{b}$ is used for significantly higher values. Biological replicates $=3$. started to form, the deposition of calcium and presence of alkaline phosphatase was evident as they stained positively for Alizarin Red S and Alkaline Phosphatase (Fig. 3). On dd 14, small opaque aggregates, covering the clusters of cells, were formed and were stained positively with Alizarin Red S and von Kossa, indicating areas of calcification (Figs. $\mathbf{3}$ and 4). At the same time new clumps of cells started to form and the cells surrounding the aggregates were stained positively for Alkaline Phosphatase. On dd 21 and 28 the opaque aggregates tended to cover $>90 \%$ of the culture dish (Fig. 4) and were stained positively with Alizarin Red $S$ and von Kossa (Figs. 3 and 4). Mineralization was also easily visible to the eye due to the opacity of the bottom of the culture flask after dd 21.

Among the biochemical markers of osteogenesis, COL1A1 (30\%) and SPARC (10\%) resulted the most abundantly expressed genes (considering also the adipogenic genes) in both cell types (Fig. 5 top panel). SPP1 percentage mRNA abundance was around $0.3-0.6 \%$, while percentage mRNA abundance of $A L P$ and BGLAP was remarkably low in both cell types (Fig. 5 top panel).

Prior to osteogenic differentiation the relative mRNA abundance of all the genes was different between ADSC and BMSC (Fig. 5 bottom panel). Transcripts of COLIAl and $S P A R C$ were more abundant in ADSC vs. BMSC, while mRNA of BGLAP and SPP1 was higher in BMSC vs. ADSC. $A L P$ tended to be $(\mathrm{P}=0.07)$ largely expressed in BMSC compared to ADSC (Fig. 5 bottom panel).

The expression patterns of ALP, COL1A1, and SPARC were very similar between them and characterized by a biphasic pattern in the BMSC, with a rapid increase starting as soon as dd 2, a peak around dd 7 (except for $A L P$, which peaked at dd 4) followed by a nadir around dd 14 (Fig. 6). In ADSC expression of these 3 genes increased through differentiation and followed the same pattern, with a peak in expression at dd 14. However, each of the 3 genes had a lower increase in expression in ADSC compared to BMSC. For SPP1 a decrease in expression was observed, for BMSC, during the first 4 days of differentiation, followed by an increase and a peak at dd 21. ADSC had a numerical decrease in expression of this gene at dd 2 and 4 followed by an increase with a peak at dd 21 (Fig. 6). Transcription of BGLAP increased only in BMSC with a peak at dd 7, while it decreased in ADSC at dd 14 (Fig. 6).

\subsection{Adipogenic Differentiation}

Morphological changes during adipogenic differentiation of ADSC and BMSC are reported in Fig. (7). The image analyses of percentage of total stained area, percentage of differentiated cells (i.e. with lipid droplets), and percentage stained area in differentiated cell are reported in Fig. (8).

As expected, ADSC on dd 0 did not stain positively for Oil Red O staining. On dd 2, ADSC changed their shape from elongated to flattened, polygonal, round or star-like, and began to grow in swirl patterns on the bottom of the flasks (Fig. 7). At this time the accumulation of lipids also began as shown by the presence of cells with lipid droplets. On dd 4 the number of differentiated cells increased along with the accumulation of lipids in the cells (Figs. 7 and 8). The percentage of area stained per differentiated cell in 


\section{No staining}
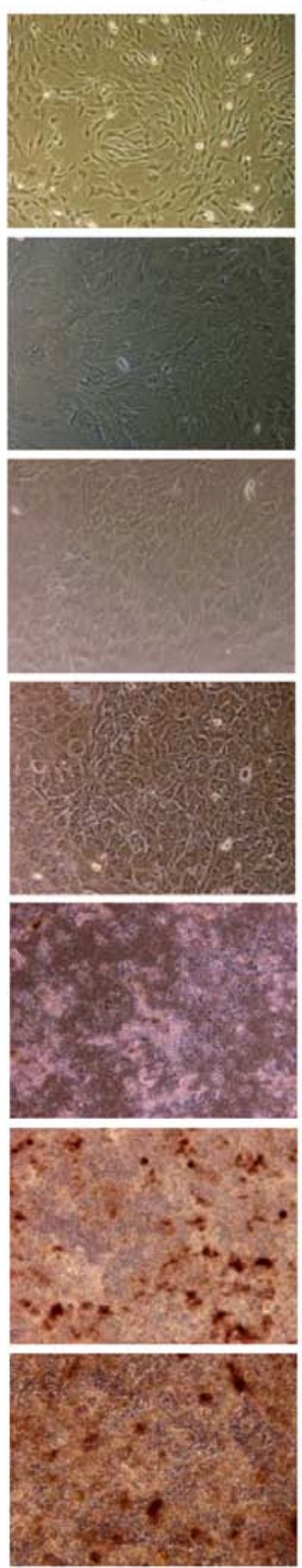

Alk. Phos.
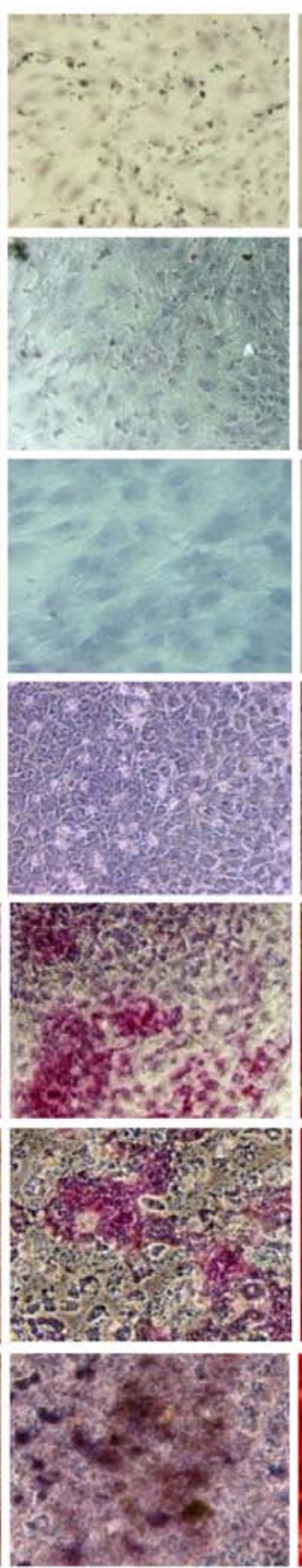

Alizarin
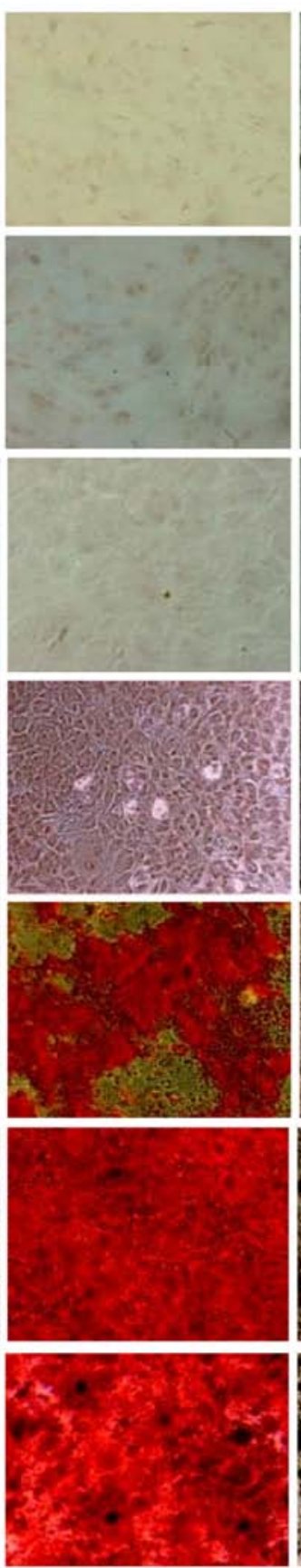

von Kossa
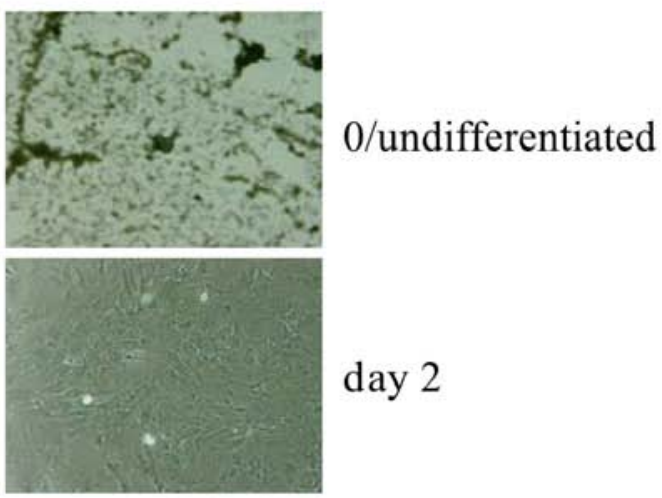

day 2

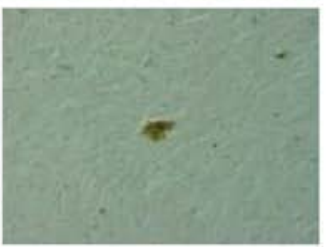

day 4

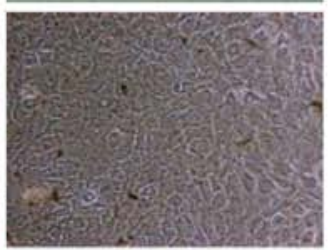

day 7

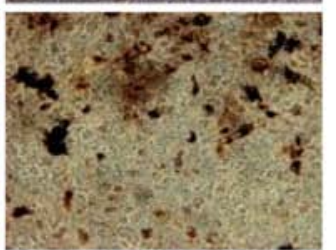

day 14

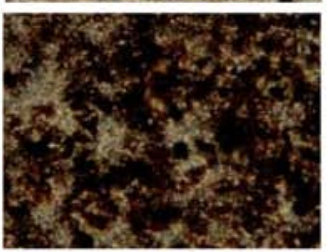

day 21

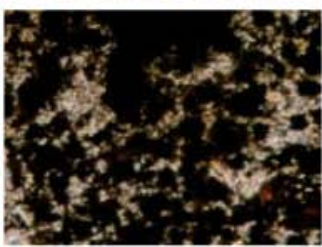

day 28

Fig. (4). Morphological changes in BMSC during osteogenic differentiation in vitro. Panels are staining for (left to right): no staining, Alkaline Phosphatase (Alk. Phos.; cells with Alk. Phos. appear red), Alizarin Red S (positive cells containing calcium deposits appear red), and von Kossa (black areas are areas of calcium deposition). Panels are days of differentiation (top to bottom): undifferentiated (day 0), and days of differentiation 2, 4, 7, 14,21, and 28. Passage 3 cells were induced to differentiate by osteogenic medium at $\sim 80 \%$ confluence. Magnification is $100 \times$ for all the pictures. Biological replicates $=3$.

ADSC increased rapidly by dd 2 and dd 4 , then was reduced at dd 7 before increasing again by dd 14 (Fig. 8). The percentage of differentiated cells increased linearly until dd 7, remained constant from dd 7 to dd 14, before increasing again between dd 14 and dd 21 (Fig. 8).

BMSC on dd 0 were fibroblast-like cells, which did not stain positively for Oil Red O (Figs. 7 and 8). After dd 2, BMSC changed their shape from elongated to polygonal, round or star shaped. Even though morphological changes were seen, there was no accumulation of fat droplets, which was confirmed by the absence of Oil Red O staining (Fig. 8). On dd 4, accumulation of fat droplets was observed in a few cells (around $8 \%$ of differentiated cells), which already had accumulated a large number of small lipid droplets $\left(>4 \mu \mathrm{M}^{2}\right.$ of stained area/differentiated cell; Fig. 8). Between dd 4 and 7 the percentage of differentiated BMSC did not increase, but a large increase was observed between dd 7 and dd 14 

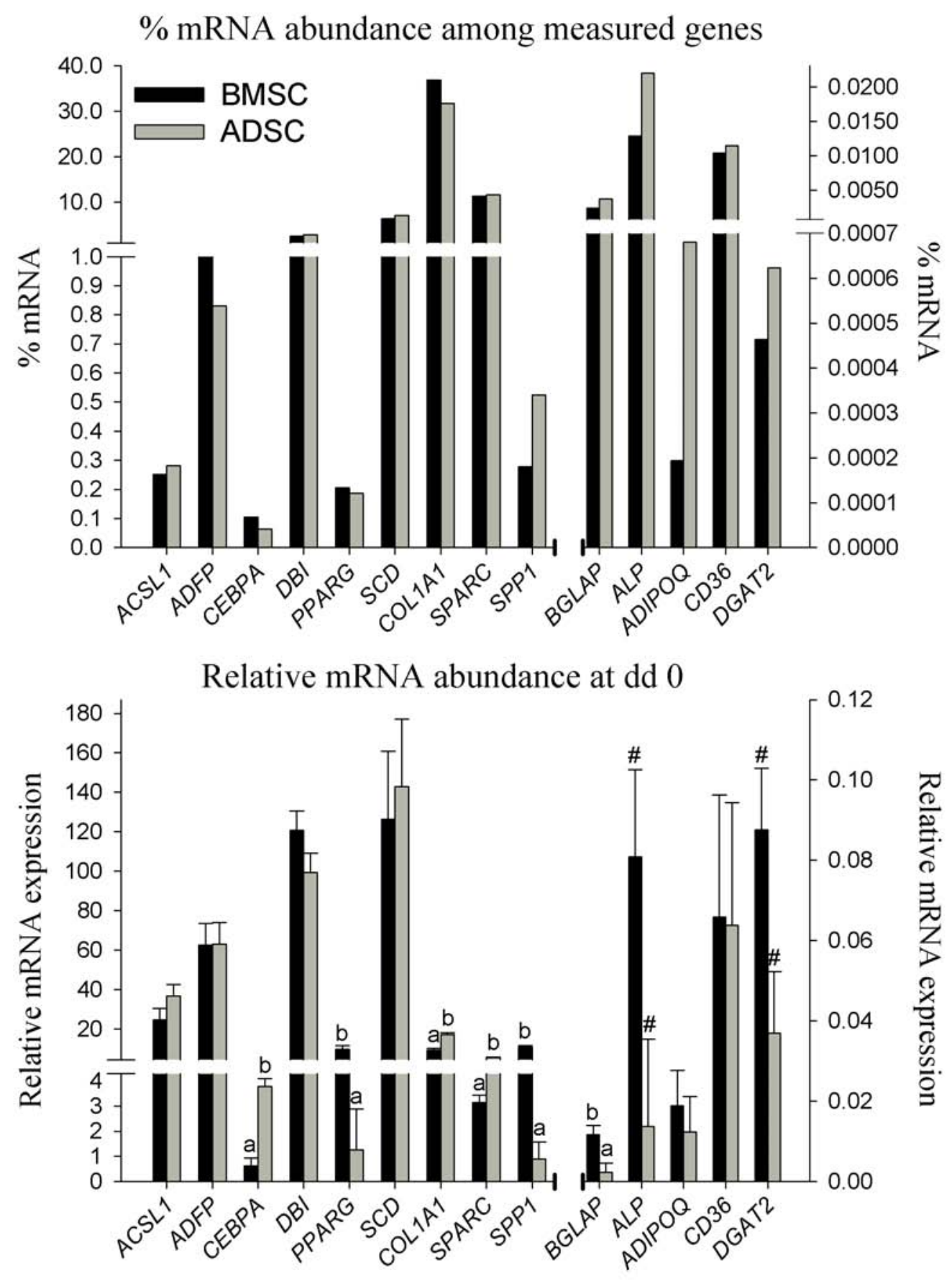

Fig. (5). Comparison of mRNA abundance of selected genes in ADSC and BMSC. Top panel: median percentage (\%) mRNA abundance among measured genes in ADSC and BMSC during osteogenic and adipogenic differentiation in vitro. Percentage of mRNA was calculated

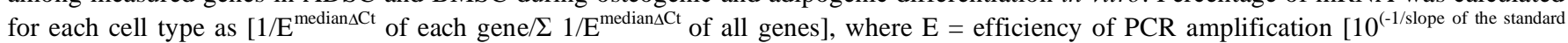
${ }^{\text {curve) }}$ ]. Bottom panel: relative mRNA abundance at differentiation day 0 in ADSC and BMCS (i.e. prior to differentiation; $\mathrm{n}=3$ for each cell type). mRNA abundance was obtained using the 7-point standard curve (see Materials and Methods). Superscripts denote: \#,\# $\mathrm{P}<0.10$ between cell types; ${ }^{\mathrm{a}, \mathrm{b}}$ is $\mathrm{P}<0.05$ between cell types. ${ }^{\mathrm{a}}$ is used for significantly lower values, while ${ }^{\mathrm{b}}$ is used for significantly higher values.

(Fig. 8). Temporal increase in lipid droplets formation and increase in percentage of differentiated cells continued through dd 28 in BMSC (Fig. 8). However, the magnitude of lipid accumulation per differentiated cell (i.e. $\mu \mathrm{M}^{2}$ stained area/differentiated cell) was lower in BMSC compared to ADCS until dd 21. At dd 28 no differences were observed but there was a lower percentage of BMSC differentiated in adipocytes compared to ADSC (Fig. 8).
Among the lipogenic genes measured, $S C D(\sim 6 \%$ mRNA among all measured genes) and $D B I(\sim 2.5 \%)$ were the most abundant in both cell types, while $A D I P O Q$ and DGAT2 were the least abundant (Fig. 5, top panel).

The relative mRNA abundance of all the lipogenic genes at dd 0 was similar between the ADSC and BMSC, except for the transcription factors $P P A R G$ and $C E B P A$ (Fig. 5, 


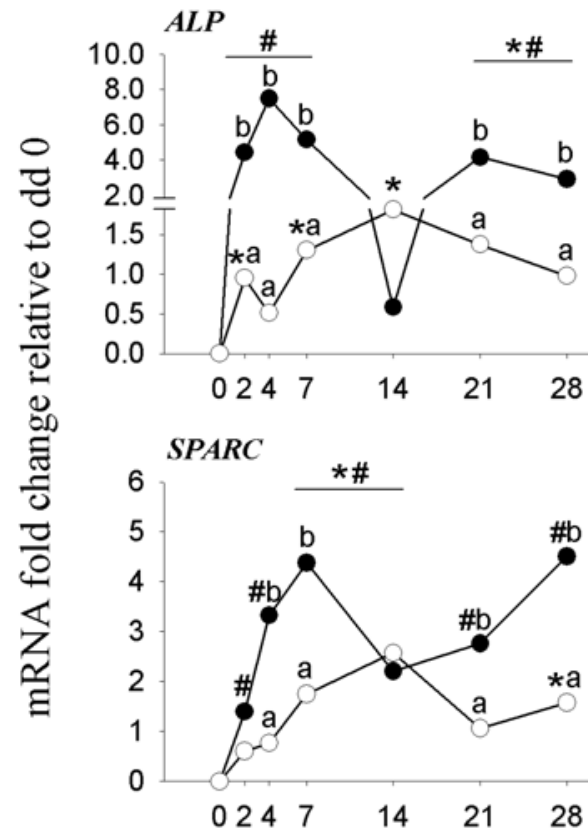

Day of differentiation

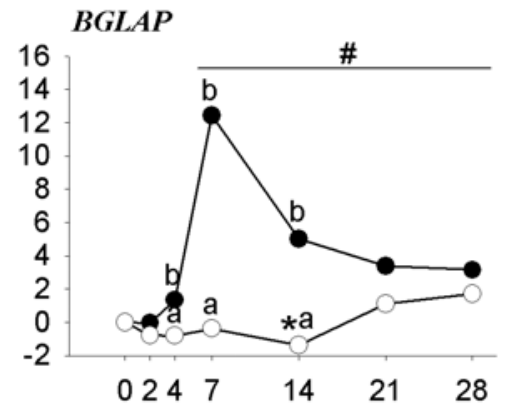

SPP1

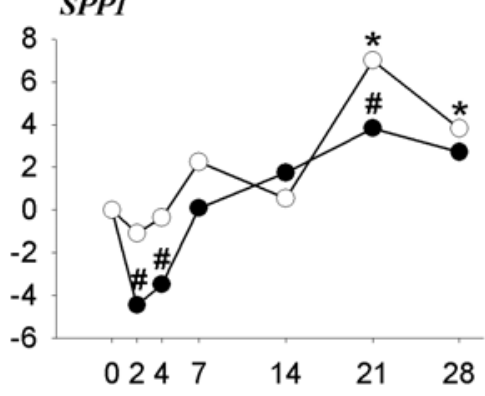

Day of differentiation

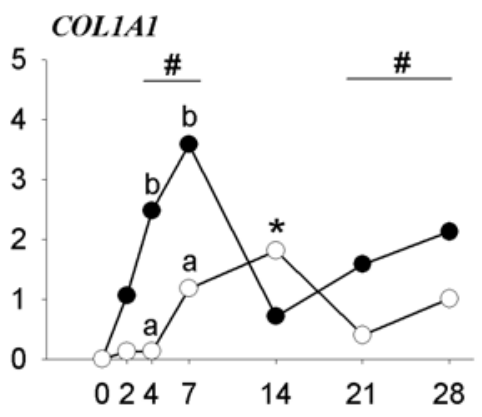

Day of differentiation

Fig. (6). Pattern of mRNA abundance for genes in ADSC and BMSC during osteogenic differentiation in vitro. Data are expressed as $n$-fold change relative to differentiation day (dd) 0 for osteogenic marker genes ALP, BGLAP, COL1A1, SPARC, and SPP1. Superscripts denote: ${ }^{*, \#}$ is $\mathrm{P}<0.05$ at each time point vs. dd 0 in ADSC and BMSC, respectively; ${ }^{\text {a }}$ b is $\mathrm{P}<0.05$ between cell types at individual time points. ${ }^{a}$ is used for significantly lower values, while ${ }^{\mathrm{b}}$ is used for significantly higher values. The overall effect of time, cell type, and time $\times$ cell type was significant $(\mathrm{P}<0.05)$ for all genes except time $\times$ cell type on $S P P 1(\mathrm{P}>0.20)$. Biological replicates $=3$.

day 0

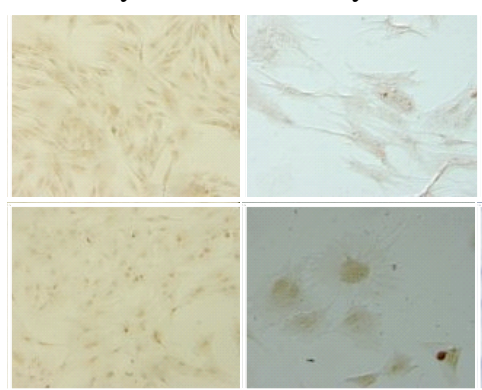

day 4

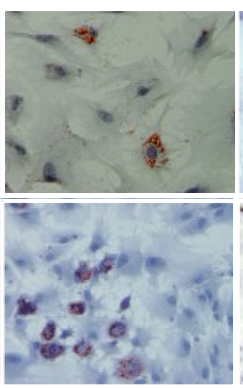

day 7

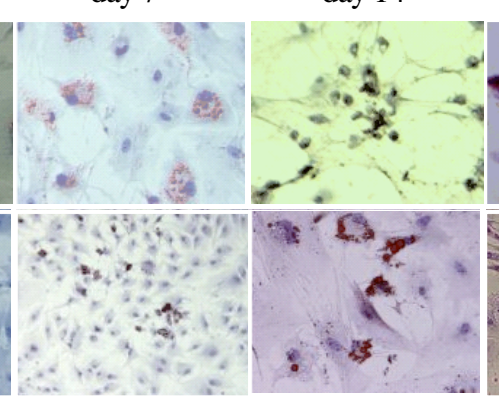

day 21

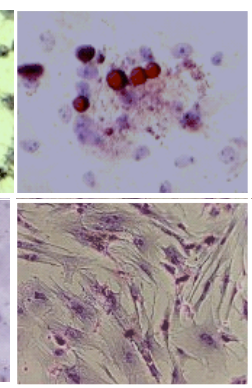

day 28

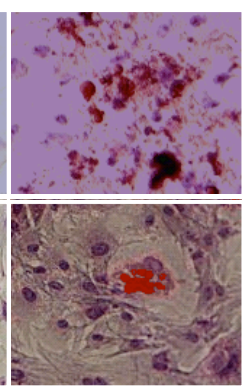

Fig. (7). Morphological changes in ADSC and BMSC during adipogenic differentiation in vitro. Oil Red O staining of ADSC and BMSC from differentiation day 0 to 28 . Magnification is $100 \times$ for day 0 ADSC and BMSC and day 7 BMSC; and $400 \times$ for all the other pictures. Biological replicates $=3$.

bottom panel). Expression of PPARG was higher and CEBPA was lower in BMSC compared to ADSC. DGAT2 tended to be largely expressed in BMSC compared to ADSC (Fig. 5, bottom panel).

During the adipogenic differentiation, $A C S L 1, S C D, D B I$, and $A D I P O Q$ had analogous temporal expression patterns among the two cell types, with a significant increase after dd 4 (Fig. 9). Expression patterns of $A D F P$ and $C D 36$ increased as soon as dd 2 , and were elevated throughout differentiation in both cell types; however, ADSC had a higher expression of these genes compared to BMSC (Fig. 9). The mRNA expression of DGAT2 increased for both cell types after dd 4, with a larger increase in ADSC vs. BMSC at dd 14 and 28. The temporal increase in expression of CEBPA was remarkably higher for all the time points in BMSC compared to ADSC, and the opposite was observed for PPARG (Fig. 9). When normalized data nontransformed as a fold change were considered, it was evident that BMCS had more relative mRNA for PPARG and lower mRNA for CEBPA throughout differentiation (Fig. S5), despite the lower fold change in expression of $P P A R G$ and the greater fold change in expression of $C E B P A$.

\section{DISCUSSION}

The goal of this study was to provide a morphological and transcriptomic comparison of porcine ADSC and BMSC as they differentiated in vitro towards the osteogenic and adipogenic lineages. By comparing ADSC and BMSC, the present study aimed to examine the possibility of using ADSC as an alternative source of stem cells for therapeutic 

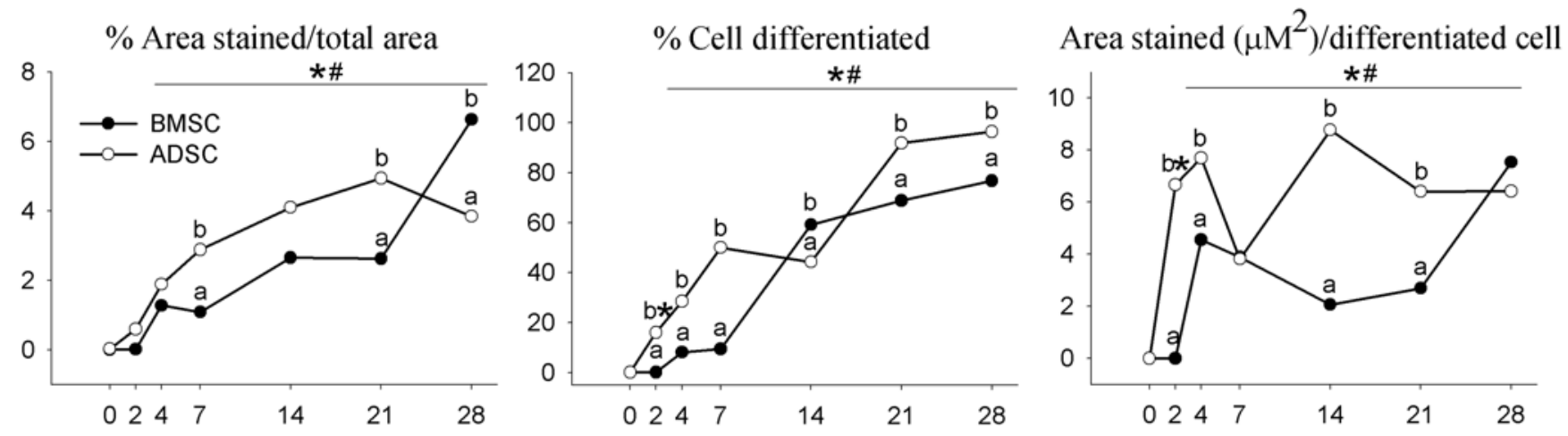

\section{Day of differentiation}

Fig. (8). Oil Red $O$ staining assessments in ADSC and BMSC during adipogenic differentiation in vitro. Oil Red O staining image analysis from dd 0 to 28 . Graphs are: the percentage (\%) area stained/total area analyzed; percentage (\%) of cells differentiated (i.e. cells with presence of at least one lipid droplet); and area stained/ differentiated cell, which is an estimate of lipid accumulation in each differenti-

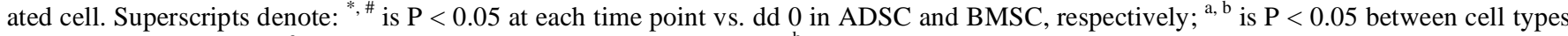
at individual time points. ${ }^{\mathrm{a}}$ is used for significantly lower values, while ${ }^{\mathrm{b}}$ is used for significantly higher values. Biological replicates $=3$.
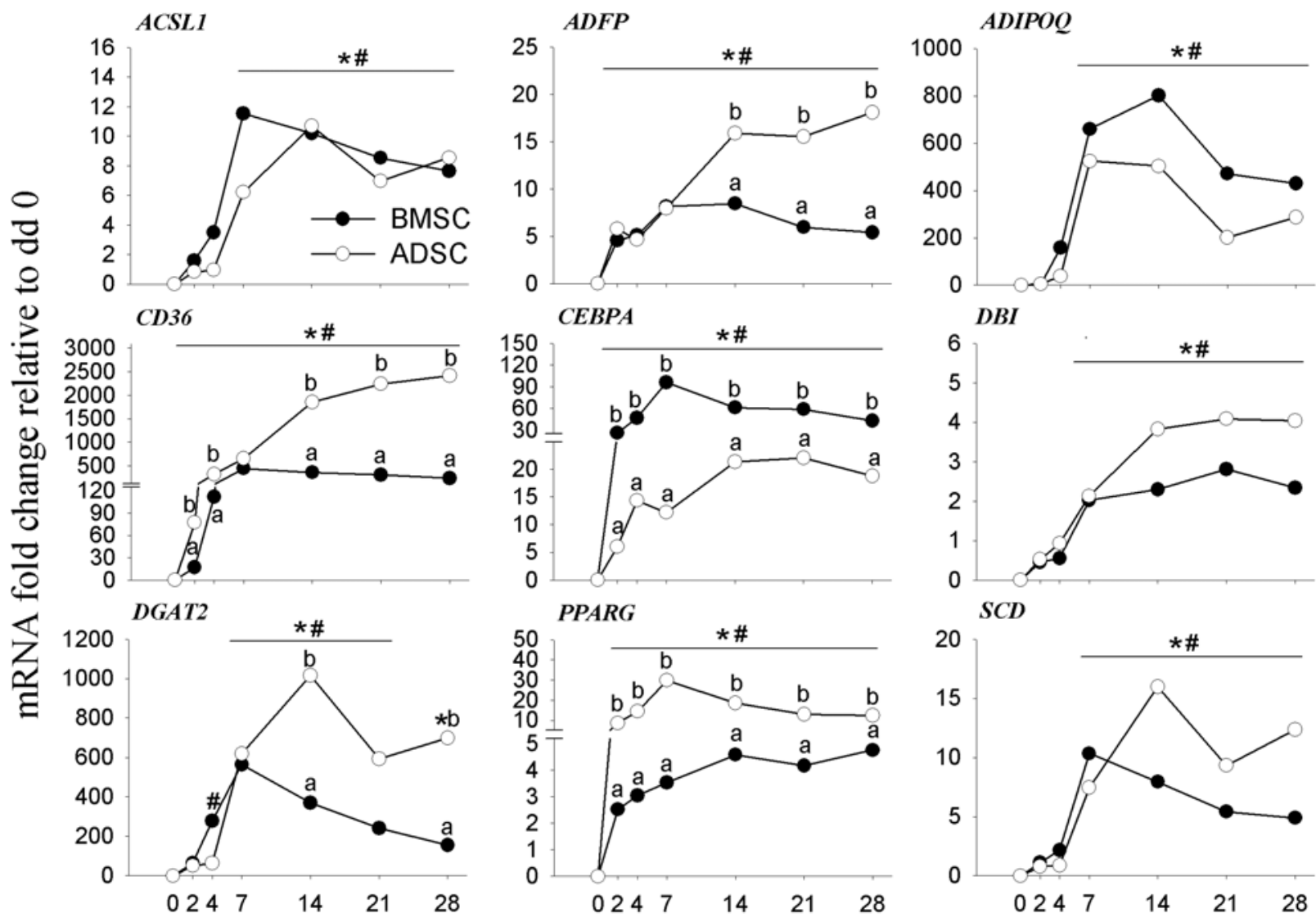

Day of differentiation

Fig. (9). Pattern of mRNA abundance for genes in ADSC and BMSC during adipogenic differentiation in vitro. Data are expressed as fold change relative to differentiation day (dd) 0 for adipogenic marker genes: ACSL1, ADFP, ADIPOQ, CD36, CEBPA, DBI, DGAT2, $P P A R G$ and SCD. Superscripts denote: ${ }^{*} \#$ is $\mathrm{P}<0.05$ at each time point vs. dd 0 in ADSC and BMSC, respectively; ${ }^{\mathrm{a}, \mathrm{b}}$ is $\mathrm{P}<0.05$ between cell types at specific time point. ${ }^{\mathrm{a}}$ is used for significantly lower values, while ${ }^{\mathrm{b}}$ is used for significantly higher values. Overall time effect was significant $(\mathrm{P}<0.05)$ for all genes; overall cell type effect was significant $(\mathrm{P}<0.05)$ for all genes except $S C D$ and tendency was observed for ACSL1 $(\mathrm{P}=0.06)$ and ADIPOQ $(\mathrm{P}=0.06)$; overall time $\times$ cell type effect was significant $(\mathrm{P}<0.05)$ for $A D F P, C D 36, C E B P A$, $D G A T 2$, and $P P A R G$. Biological replicates $=3$.

purposes in humans. In fact, adipose presents several advantages vs. bone marrow as tissue source. Compared to bone marrow adipose tissue has a larger number of stem cells, it is much more abundant in the body, and it is easy to harvest without leaving post-surgical deformities [35]. 
In this study, porcine adult stem cells harvested from both bone marrow and adipose tissue were able to attach to tissue culture dishes and form adherent colonies in vitro. The number of $\mathrm{CFU}$ in BMSC $(0.07 \pm 0.01 \%)$ was much lower than the number of CFU in $\operatorname{ADSC}(0.39 \pm 0.02 \%)$. In humans, Kern et al. obtained $0.05 \% \mathrm{CFU}$ in ADSC and $0.008 \%$ $\mathrm{CFU}$ in BMSC [36]. The larger number of CFU that we obtained, in both cell types, may be due to species differences. Moreover, the age of the pigs used in the present study (6 months) and the age of the patients (44-84 years) in the study of Kern et al. might have influenced the relative number of CFU obtained from the BMSC. In fact, the number of adherent BMSC cells, and consequentially the number of CFU, decreases with increasing age [37]. Furthermore, in the present study the cells were recovered from the femur of the animals and not aspirated from the iliac crest as it was done in some of the patients in Kern et al. [36].

\subsection{Osteogenic Differentiation}

Both ADSC and BMSC differentiated along the osteogenic lineage albeit with some differences. During the first 4 days of differentiation, both cell types went through the same morphological changes, which started as early as dd 2 (Figs. 2 and 4). However, from dd 7 the two cell types had different morphological changes. Concomitantly, accumulation of calcium started, initially higher in ADSC compared to BMSC, but as differentiation progressed, it became greater in BMSC (Fig. 3). The increase in expression of most osteogenic genes was observed, as early as dd 2 (i.e. $A L P$ ), in both cell types and they had greater expression in BMSC vs. ADSC, except SPPl (Fig. 6).

At dd 2 morphological changes were also seen in human [37, 38], chicken [39], and rat cells [40]. However, our results differ from what was seen in porcine ADSC by Qu et al. [41], where morphological changes in the cell structure were observed only after 5 days in culture. In the present study, in addition to the visual observation of the cells, we measured the relative mRNA abundance of specific osteogenic genes by RT-PCR during the entire differentiation. This allowed us to identify the onset of osteogenic differentiation to be as early as dd 2 in ADSC (i.e., ALP expression), which supports the earlier morphological changes seen in our study compared to the one of Qu et al. Staining of the ADSC was observed after two weeks of osteogenic induction in the Qu et al. study. In the present study, cells started the mineralization (i.e. evidence of staining) by 1 week of induction (Fig. 3). This also is supported by the large change in expression of the osteogenic genes that we observed in both cell types after dd 4 .

For both ADSC and BMSC, the osteogenic differentiation did not happen at the same moment for all the cells, but progressively involved an increasing proportion of cells. This is an important observation because it points out that only a portion of the cells were induced to differentiate during the first days of osteogenic differentiation, while most cells needed about 2 weeks to initiate differentiation. We can speculate that both ADSC and BMSC had cells with a different sensitivity to the osteogenic medium, and we can envisage a process in which the cells that reached differentiation stimulated other cells to differentiate.
The difference in morphology, observed between the two cell types during osteogenic differentiation, is of particular interest. Large osteogenic nodules were formed by ADSC in contrast to a sheet of small osteogenic nodules which were generated by BMSC during the osteogenic differentiation. It is possible that the two cell types, in accordance with their tissue of origin (adipose tissue or bone marrow), are committed to generate different types of bone. In this regard, it is known that there are two main categories of bone: spongy bone (trabecular bone, cancellous bone) and compact bone (cortical bone). Spongy bone is composed of a network of branching bone spicules or trabeculae. The spaces between the bone spicules contain bone marrow. Compact bone appears as a mass of bony tissue lacking spaces visible to the unaided eye. We might speculate that the ADSC may have a commitment to form spongy bone, while BMSC may have a commitment towards the formation of compact bone. However, our findings require further investigation and elucidation of a potential mechanism for these differences.

The different morphological organization of the two cell types might have influenced the higher percentage stained area of BMSC vs. ADSC after dd 7. In fact the method used for the measurement of the stained area had some limitations. ImageJ program cannot account for areas of different color intensity, which parameter was very important for the quantification of ADSC osteogenic differentiation. In fact, once the ADSC nodules started to form, they were recruiting more and more cells through time, which were accumulating on top of each other. For this reason, the increase in diameter of the nodules was probably minor compared to the increase in nodule hight. Moreover, during the first two weeks of the osteogenic differentiation new nodules continued to form, so that by day 28 , besides the large defined nodules, there were few nodules of new formation, which could be in the area we randomly sampled for our measurements.

The difference in the positive histological staining in BMSC vs. ADSC observed at dd 7 (Fig. 3), suggests a delay in the BMSC osteogenic differentiation compared to ADSC. Our finding is in accordance with Ringe et al., where porcine BMSC did not show positive staining for Alkaline Phosphatase and von Kossa until day 12 of osteogenic differentiation [42]. However, the up regulation of $A L P, C O L 1 A 1$, and $S P A R C$ earlier and higher in BMSC compared to ADSC (Fig. 6), apparently seems to be in discord with the results from the osteogenic histological staining. In fact, since the percentage of stained area of Alkaline Phosphates and Alizarin Red S at dd 7 was higher in ADSC vs. BMSC, we would expect an earlier increase in expression of the osteogenic markers (ALP, COLIA1, SPARC, BGLAP, and SPPI) in ADSC than BMSC. Nevertheless, our results are in accordance with Peng et al., who showed that qPCR analysis of ADSC and BMSC from SD rats, during osteogenic differentiation, resulted in an up-regulation of osteogenic markers, such as COLIAI and BGLAP, in BMSC well ahead of ADSC [43]. An explanation for the discordance between gene expression and staining data is not apparent; however, we can infer that the increase in expression of osteogenic genes, in BMSC, was not readily translated into proteins, or that post-translational mechanisms were at play. Another possible explanation is that during the first days of osteo- 
genic differentiation in BMSC, the nodules were too small and diffuse to be detected by the staining, while in ADSC the few nodules were more aggregated and easily detectable.

Overall, the increase in ALP, COLIA1, SPARC, and $S P P 1$ in both cell types is of biological significance since those proteins are characteristic of onset of osteogenenic differentiation. They are important during matrix deposition [44-46]. In general, COL1Al is one of the earliest osteoblasts markers to be up-regulated in committed osteoprogenitors, and it seems to influence the expression of $A L P$ and SPPI [46]. $A L P$ increases in early osteogenic differentiation in osteoprogenitors, then decreases when mineralization has well progressed [46]. Our results are consistent with these previous findings. $S P P 1$ is involved in calcium binding ions and hydroxyapatite during the mineralization process [24]. Interestingly, it was down-regulated at the beginning of the differentiation, in both ADSC and BMSC, when the processes of matrix deposition were occurring, but then its expression increased, perhaps because the mineralization of the matrix began. BGLAP is a marker of mature osteoblasts and appears with bone mineralization [25, 46]. In the present study, indeed, BGLAP was minimally expressed during the first days of differentiation in BMSC and then increased rapidly to reach a peak at dd 7 when bone mineralization began, as evidenced by staining analysis (Fig. 3). However, the same gene in ADSC did not increase during the osteogenic differentiation. Together with the percentage of area stained observed for Alizarin Red S and von Kossa in ADSC, these data suggest a lesser mineralization of bone matrix and calcium deposition in this cell type compared to BMSC.

\subsection{Adipogenic Differentiation}

In the present study we observed that both ADSC and BMSC differentiated along the adipogenic lineage, although there were some differences.

The overall morphological observation of ADSC and BMSC, which had differentiated into adipogenic cells, clearly revealed the typical phenotype of adipocytic cells with a coalescence of small lipid droplets to fill the whole cytoplasm of single cells. Two days after induction, ADSC and BMSC changed from fibroblast-like cells to polygonal, round, and star shaped. ADSC not only had morphological changes, but fat droplet formation was observed. This is similar to the observations from the study of Qu et al., where by day 2 of adipogenic differentiation in porcine ADSC, the first cells that were filled with lipid droplets appeared [41].

In this study, the first lipid droplets in BMSC were observed at dd 4, whereas in Zou et al., [47] the formation of the first lipid droplets in pig BMSC was observed at day 7. The different temporal response of the BMSC in the Zou et al. study may be due to the differentiation medium used, which was basal media supplemented only with dexamethason, while in our case, the medium was enriched also by isobutylmethylxanthine, insulin, and indomethacin. Among those isobutylmethylxanthine and insulin are essential for $P P A R G$ activity $[48,49]$. The increase in activity of $P P A R G$ can drive large accumulation of lipid droplets due to its strong induction in expression of lipogenic genes [50]. This also is evident in the temporal transcription results of the adipogenic genes measured in the present experiment (Fig. 9). Moreover, the time of appearance of the first lipid drop- lets in BMSC, observed in our experiment, is similar to the time reported by Bosch et al. [51] who used the same cell type and animal species. However, in Bosch et al., the number of BMSC with lipid accumulation and the size of the lipid droplets increased until days 8-9 and then reached a plateau (days 12-14) [51], while in our study the dynamics of time of lipid accumulation and increasing size of lipid droplets was quite different. In fact, the number of cells with lipid accumulation and the size of lipid droplets, in both cell types, increased through time until the end of the 4 weeks (Fig. 8). As observed before for the osteogenic differentiation, this is interesting because it suggests that the adipogenic differentiation, for both cell types, did not happen simultaneously in all the cells, but rather occurred gradually and involved more and more cells. Only part of the cells differentiated during the first days of adipogenic differentiation, while most of the cells needed as much as 2 weeks to initiate differentiation. We can speculate that both ADSC and BMSC had cells with a different sensitivity to the adipogenic medium, and we can speculate a process in which the cells that reached differentiation stimulated other cells to differentiate.

All the measured genes were markers of adipogenesis, some of the adipogenesis per se (i.e., PPARG and CEBPA), and others of lipogenesis and lipid droplets formation (i.e., $D G A T 2$ and $A D F P$ ). All those genes increased significantly in both cell types, which thing strongly supports a differentiation in adipocytes of both mesenchymal stem cells. Most of the adipogenic genes had a similar expression pattern between the two cell types, especially during the initial days of the differentiation (dd 0 through dd 7; Fig. 9). The high increase in expression patterns of CD36, ACSL1, and DBI observed suggests a large influx, activation, and intracellular transport of free fatty acids during adipogenesis in both cell types. The expression of $S C D, D G A T 2$, and $A D F P$, genes involved in triglyceride synthesis and lipid droplets formation, increased significantly in both cells during adipogenesis. However, after 7 days of differentiation, the expression patterns of DGAT2 and ADFP became greater in ADSC versus BMSC, suggesting a greater lipid droplet formation in the former, which agreed with observed accumulation of triglycerides measured by Oil Red $\mathrm{O}$ histological staining in ADSC.

One interesting observation was that of lipid droplet formation, already evident at dd 2 in ADSC, while in BMSC they were detectable only from dd 4 despite very similar relative mRNA abundance at dd 0 (Fig. 5), and a similar increase in expression of almost all the lipogenic genes measured during early differentiation (i.e., until dd 7; Fig. 9). One exception was $C D 36$ which had a greater increase in expression in ADSC compared to BMSC, at the beginning and throughout all the differentiation, suggesting that the uptake of preformed fatty acids is crucial for lipogenesis and adipocyte maturation.

The $A D I P O Q$ expression pattern is indicative of mature adipocytes, and the high similarity among the two cell populations for the expression of this gene, underlines a similar capacity to differentiate towards adipocites for both ADSC and BMSC.

It has been clearly demonstrated that CEBPA is expressed just before the transcription of most adipocyte- 
specific genes is initiated [50], as well as PPARG is induced concomitantly with and prior to the transcriptional activation of most genes involved in lipogenesis [50]. In this regard, it is interesting that the temporal expression of $C E P B A$ and $P P A R G$ was characterized by a sharp, large increase as soon as dd 2, which also occurred before the increase in expression of the other lipogenic genes in the present study (Fig. 9). Previous findings have proved that $C E B P A$ is both required and sufficient to induce adipocyte differentiation [52, 53]. PPARG is the most adipose-specific of the PPAR isoforms. It is expressed at the highest levels in adipose tissue and adipocyte cell lines, and at low levels, or not at all, in other tissues and cell lines [54, 55]. Moreover, CEBPA and $P P A R G$ cooperate in inducing the expression of many adipocyte-specific proteins implicated in generating and maintaining the adipocyte phenotype [56, 57]. In the present study, the greater relative mRNA abundance of PPARG in BMSC, compared to ADSC (Fig. 5), was partly unexpected since transcripts of PPARG are abundant in adipocytes and we would have hypothesized a higher expression in ADSC due to the tissue of origin. Moreover, the induction in expression measured as fold change of PPARG and CEBPA was different between the two cell types. The larger temporal increase in expression was observed in both cells for the genes with original (i.e., dd 0) lower mRNA abundance (Figs. 5 and 9). This differential expression allowed obtaining a similar mRNA abundance of the two genes, in both cell types, during adipogenesis. Overall our data are supportive of a cooperation between $C E B P A$ and $P P A R G$ during adipogenesis as previously reported [50].

\section{CONCLUSION}

In conclusion, despite the ability of both cell types to differentiate towards the osteogenic and adipogenic lineages, our observations revealed specific morphological and transcriptomic differences between ADSC and BMSC. Nevertheless, those differences might be overcome by a better control of the differentiation process. The possibility of using ADSC as a substituted of BMSC for human therapies requires a better understanding of the molecular events underlining the differentiation steps. In this regard, evaluation in more details of molecular mechanisms controlling differentiation using high throughput transcriptomics by microarrays and other cellular measurements, such as migration capacity, can provide new insights. Finally, our findings require further investigation and elucidation.

\section{ACKNOWLEDGEMENTS}

We would like to thank Synthia Lane, Tara VanEtten, Dongshin Kim, Cristiano Feltrin, and Glenn Bressner for technical assistance, sample collection and animal care. This work was supported by a grant from the Illinois Regenerative Medicine Institute, \#IDPH 63080017.

\section{SUPPORTIVE/SUPPLEMENTARY MATERIAL}

Supplementary material can be viewed at: http://www. bentham.org/open/totermj

A supplementary materials file is available online which contains additional description of materials and methods for histological staining for osteogenic and adipogenic differentiation, quantitative Real-Time RT-PCR (qPCR) analysis, primers design and evaluation, selection and evaluation of the internal control genes, quantification of histological staining by means of ImageJ software.

The file contains 3 tables and 6 figures:

Tables S1 reports features of the primer-pairs used.

Table S2 and Table S3 report sequencing results of primerpairs.

Fig. (S1) reports potential co-regulation among tested internal control genes.

Figs. (S2 and S3) report the evaluation of internal control genes for qPCR normalization.

Figs. (S4 and S5) report original relative mRNA abundance (i.e., not transformed in fold change) for the 5 osteogenic and 9 adipogenic gene markers used in the paper.

Fig. (S6) reports the radius calculated in Alizarin Red S stained nodules during osteogenic differentiation in ADSC.

\section{REFERENCES}

[1] Stamm C, Kleine HD, Choi YH, et al. Intramyocardial delivery of CD133+ bone marrow cells and coronary artery bypass grafting for chronic ischemic heart disease: safety and efficacy studies. J Thorac Cardiovasc Surg 2007; 133: 717-25.

[2] Horwitz EM, Gordon PL, Koo WK, et al. Isolated allogeneic bone marrow-derived mesenchymal cells engraft and stimulate growth in children with osteogenesis imperfecta: Implications for cell therapy of bone. Proc Natl Acad Sci U S A 2002; 99: 8932-7.

[3] Aggarwal S, Pittenger MF. Human mesenchymal stem cells modulate allogeneic immune cell responses. Blood 2005; 105: 1815-22.

[4] Kassem M, Mosekilde L, Eriksen EF. 1,25-dihydroxyvitamin D3 potentiates fluoride-stimulated collagen type I production in cultures of human bone marrow stromal osteoblast-like cells. J Bone Miner Res 1993; 8: 1453-8.

[5] Justesen J, Stenderup K, Eriksen EF, Kassem M. Maintenance of osteoblastic and adipocytic differentiation potential with age and osteoporosis in human marrow stromal cell cultures. Calcif Tissue Int 2002; 71: 36-44.

[6] Zhang FB, Yang HT. Plasticity of bone marrow mesenchymal stem cells differentiating into cardiomyocytes and the potential of cardiac therapeutics. Sheng Li Ke Xue Jin Zhan 2006; 37: 199-204.

[7] Johnstone B, Hering TM, Caplan AI, Goldberg VM, Yoo JU. In vitro chondrogenesis of bone marrow-derived mesenchymal progenitor cells. Exp Cell Res 1998; 238: 265-72.

[8] Lei Z, Yongda L, Jun M, et al. Culture and neural differentiation of rat bone marrow mesenchymal stem cells in vitro. Cell Biol Int 2007; 31: 916-23.

[9] Kiss J, Urban VS, Dudics V, Vas V, Uher F. Mesenchymal stem cells and the immune system--immunosuppression without drugs? Orv Hetil 2008; 149: 339-46.

[10] Friedenstein AJ, Gorskaja JF, Kulagina NN. Fibroblast precursors in normal and irradiated mouse hematopoietic organs. Exp Hematol 1976; 4: 267-74.

[11] Zuk PA, Zhu M, Mizuno H, et al. Multilineage cells from human adipose tissue: implications for cell-based therapies. Tissue Eng 2001; 7: 211-28.

[12] Zuk PA, Zhu M, Ashjian P, et al. Human adipose tissue is a source of multipotent stem cells. Mol Biol Cell 2002; 13: 4279-95.

[13] Fraser JK, Wulur I, Alfonso Z, Hedrick MH. Fat tissue: an underappreciated source of stem cells for biotechnology. Trends Biotechnol 2006; 24: 150-4.

[14] Ortega JJ, Diaz de Heredia C, Olive T, et al. Allogeneic and autologous bone marrow transplantation after consolidation therapy in high-risk acute myeloid leukemia in children. Towards a riskoriented therapy. Haematologica 2003; 88: 290-9.

[15] Yoshimura K, Shigeura T, Matsumoto D, et al. Characterization of freshly isolated and cultured cells derived from the fatty and fluid portions of liposuction aspirates. J Cell Physiol 2006; 208: 64-76.

[16] Gimble JM, Katz AJ, Bunnell BA. Adipose-derived stem cells for regenerative medicine. Circ Res 2007; 100: 1249-60. 
[17] Bredeson C, Leger C, Couban S, et al. An evaluation of the donor experience in the canadian multicenter randomized trial of bone marrow versus peripheral blood allografting. Biol Blood Marrow Transplant 2004; 10: 405-14.

[18] Kozlik-Feldmann R, Lang N, Aumann R, et al. Patch closure of muscular ventricular septal defects with a new hybrid therapy in a pig model. J Am Coll Cardiol 2008; 51: 1597-603.

[19] Saunte DM, Hasselby JP, Brillowska-Dabrowska A, et al. Experimental guinea pig model of dermatophytosis: a simple and useful tool for the evaluation of new diagnostics and antifungals. Med Mycol 2008; 46: 303-13.

[20] Alam HB, Duggan M, Li Y, et al. Putting life on hold-for how long? Profound hypothermic cardiopulmonary bypass in a Swine model of complex vascular injuries. J Trauma 2008; 64: 912-22.

[21] Rogatcheva MB, Fritz KL, Rund LA, et al. Characterization of the porcine ATM gene: towards the generation of a novel non-murine animal model for Ataxia-Telangiectasia. Gene 2007; 405: 27-35.

[22] Kuca K, Jun D. Reactivation of sarin-inhibited pig brain acetylcholinesterase using oxime antidotes. J Med Toxicol 2006; 2: 141-6.

[23] Bionaz M, Loor JJ. ACSL1, AGPAT6, FABP3, LPIN1, and SLC27A6 are the most abundant isoforms in bovine mammary tissue and their expression is affected by stage of lactation. J Nutr 2008; 138: 1019-24.

[24] Guo L, Kawazoe N, Hoshiba T, et al. Osteogenic differentiation of human mesenchymal stem cells on chargeable polymer-modified surfaces. J Biomed Mater Res A 2008; 87: 903-12.

[25] Rath B, Nam J, Knobloch TJ, Lannutti JJ, Agarwal S. Compressive forces induce osteogenic gene expression in calvarial osteoblasts. J Biomech 2008; 41: 1095-103.

[26] Denhardt DT, Guo X. Osteopontin: a protein with diverse functions. FASEB J 1993; 7: 1475-82.

[27] Jiang HP, Serrero G. Isolation and characterization of a full-length cDNA coding for an adipose differentiation-related protein. Proc Natl Acad Sci U S A 1992; 89: 7856-60.

[28] Bartz R, Zehmer JK, Zhu M, et al. Dynamic activity of lipid droplets: protein phosphorylation and GTP-mediated protein translocation. J Proteome Res 2007; 6: 3256-65.

[29] Rosen ED. The transcriptional basis of adipocyte development. Prostaglandins Leukot Essent Fatty Acids 2005; 73: 31-4.

[30] Lobo S, Bernlohr DA. Fatty acid transport in adipocytes and the development of insulin resistance. Novartis Found Symp 2007; 286: 113-21; discussion 21-6, 62-3, 96-203

[31] Nicholson AC, Hajjar DP, Zhou X, et al. Anti-adipogenic action of pitavastatin occurs through the coordinate regulation of PPARgamma and Pref-1 expression. Br J Pharmacol 2007; 151: 807-15.

[32] Fisher E, Nitz I, Gieger C, et al. Association of acyl-CoA-binding protein (ACBP) single nucleotide polymorphisms and type 2 diabetes in two German study populations. Mol Nutr Food Res 2007; 51: 178-84.

[33] Frick F, Hume R, Robinson IC, Eden S, Oscarsson J. Hepatic and Adipose Tissue Depot-Specific Changes in Lipid Metabolism in Late-Onset Obese (LOB) Rats. Lipids 2008; 43: 313-24.

[34] Ruifrok AC, Johnston DA. Quantification of histochemical staining by color deconvolution. Anal Quant Cytol Histol 2001; 23: 291-9.

[35] Patrick CW, Jr. Tissue engineering strategies for adipose tissue repair. Anat Rec 2001; 263: 361-6.

[36] Kern S, Eichler H, Stoeve J, Kluter H, Bieback K. Comparative analysis of mesenchymal stem cells from bone marrow, umbilical cord blood, or adipose tissue. Stem Cells 2006; 24: 1294-301.

[37] D'Ippolito G, Schiller PC, Ricordi C, Roos BA, Howard GA. Agerelated osteogenic potential of mesenchymal stromal stem cells from human vertebral bone marrow. J Bone Miner Res 1999; 14: 1115-22.
[38] Bruder SP, Jaiswal N, Haynesworth SE. Growth kinetics, selfrenewal, and the osteogenic potential of purified human mesenchymal stem cells during extensive subcultivation and following cryopreservation. J Cell Biochem 1997; 64: 278-94.

[39] Kamalia N, McCulloch CA, Tenebaum HC, Limeback H. Dexamethasone recruitment of self-renewing osteoprogenitor cells in chick bone marrow stromal cell cultures. Blood 1992; 79: 320-6.

[40] Bellows CG, Aubin JE, Heersche JN. Initiation and progression of mineralization of bone nodules formed in vitro: the role of alkaline phosphatase and organic phosphate. Bone Miner 1991; 14: 27-40.

[41] Qu CQ, Zhang GH, Zhang LJ, Yang GS. Osteogenic and adipogenic potential of porcine adipose mesenchymal stem cells. In Vitro Cell Dev Biol Anim 2007; 43: 95-100.

[42] Ringe J, Kaps C, Schmitt B, et al. Porcine mesenchymal stem cells. Induction of distinct mesenchymal cell lineages. Cell Tissue Res 2002; 307: 321-7.

[43] Peng L, Jia Z, Yin X, et al. Comparative Analysis of Mesenchymal Stem Cells from Bone Marrow, Cartilage and Adipose Tissue. Stem Cells Dev 2008; 17: 761-73.

[44] Aronow MA, Gerstenfeld LC, Owen TA, et al. Factors that promote progressive development of the osteoblast phenotype in cultured fetal rat calvaria cells. J Cell Physiol 1990; 143: 213-21.

[45] Bellows CG, Heersche JN, Aubin JE. Inorganic phosphate added exogenously or released from beta-glycerophosphate initiates mineralization of osteoid nodules in vitro. Bone Miner 1992; 17: 15-29.

[46] Aubin JE. Regulation of osteoblast formation and function. Rev Endocr Metab Disord 2001; 2: 81-94.

[47] Zou L, Zou X, Chen L, et al. Multilineage differentiation of porcine bone marrow stromal cells associated with specific gene expression pattern. J Orthop Res 2008; 26: 56-64.

[48] Prusty D, Park BH, Davis KE, Farmer SR. Activation of MEK/ERK signaling promotes adipogenesis by enhancing peroxisome proliferator-activated receptor gamma (PPARgamma) and C/EBPalpha gene expression during the differentiation of 3T3-L1 preadipocytes. J Biol Chem 2002; 277: 46226-32.

[49] Schoonjans K, Martin G, Staels B, Auwerx J. Peroxisome proliferator-activated receptors, orphans with ligands and functions. Curr Opin Lipidol 1997; 8: 159-66.

[50] Mandrup S, Lane MD. Regulating adipogenesis. J Biol Chem 1997; 272: 5367-70.

[51] Bosch P, Pratt SL, Stice SL. Isolation, characterization, gene modification, and nuclear reprogramming of porcine mesenchymal stem cells. Biol Reprod 2006; 74: 46-57.

[52] Lin FT, Lane MD. CCAAT/enhancer binding protein alpha is sufficient to initiate the 3T3-L1 adipocyte differentiation program. Proc Natl Acad Sci U S A 1994; 91: 8757-61.

[53] Wang ND, Finegold MJ, Bradley A, et al. Impaired energy homeostasis in C/EBP alpha knockout mice. Science 1995; 269: 1108-12.

[54] Forman BM, Tontonoz P, Chen J, et al. 15-Deoxy-delta 12, 14prostaglandin $\mathrm{J} 2$ is a ligand for the adipocyte determination factor PPAR gamma. Cell 1995; 83: 803-12.

[55] Schoonjans K, Staels B, Auwerx J. The peroxisome proliferator activated receptors (PPARS) and their effects on lipid metabolism and adipocyte differentiation. Biochim Biophys Acta 1996; 1302 93-109.

[56] Shao D, Lazar MA. Peroxisome proliferator activated receptor gamma, CCAAT/enhancer-binding protein alpha, and cell cycle status regulate the commitment to adipocyte differentiation. J Biol Chem 1997; 272: 21473-8.

[57] Rosen ED, MacDougald OA. Adipocyte differentiation from the inside out. Nat Rev Mol Cell Biol 2006; 7: 885-96. 